\title{
MAGNETIC RESONANCE IMAGING OF THE INTERNALLY DERANGED KNEE JOINT - A PROSPECTIVE STUDY
}

Rangappa Hanumappa Chavadaki ${ }^{1}$, Praveen Kumar Reddy P2, Reneesh Uruniyan Paramban³, Swetha Gurram$^{4}$

\section{HOW TO CITE THIS ARTICLE:}

Rangappa Hanumappa Chavadaki, Praveen Kumar Reddy P, Reneesh Uruniyan Paramban, Swetha Gurram. "MRI of the internally deranged knee joint. A Prospective study". Journal of Evolution of Medical and Dental Sciences 2013; Vol. 2, Issue 42, October 21; Page: 8186-8206.

ABSTRACT: BACKGROUND \& OBJECTIVE: Trauma to knee joint is a significant cause of morbidity in the young, active individuals and athletes. An accurate diagnosis regarding the type and extent of injuries is essential for early operative as well as non-operative treatment. The most widely used diagnostic modalities to assess the joint in jury are arthroscopy and MRI. Arthroscopy, though accurate, is invasive and can cause complications. Magnetic resonance imaging (MRI) has now been accepted as the best imaging modality for non-invasive evaluation of knee injuries. This study was undertaken to study the types and incidence of injuries in traumatic knee joint by MR imaging and to compare with arthroscopy findings in selected cases. METHODS: DESIGN: Prospective study Setting: In a tertiary care hospital, symptomatic patients who were referred from various service referral hospitals and units with history of trauma and clinical suspicion of IDK were referred to department of Orthopedics, Navodaya Medical College, Raichur. Patients: 50 consecutive patients with suspicion of IDK following knee trauma were selected for the study from 2011 to 2013. INVESTIGATIONS: Clinical examinations, MRI knee (within 4 days of referral) \& in selected cases arthroscopy were performed and the findings were compared. Main Outcome Measures: Study of spectrum of MRI findings in traumatic knee and correlation of MRI findings with arthroscopy/surgical findings in selected cases. RESULTS: Of the total 50 patients evaluated, most common injury was ACL tear of which complete tears were more common. PCL tears were less common. Among the Meniscal injuries, MM tears are more common than LM and grade 2 tears are more common in both. MCL tears outnumbered LCL tears and grade 1 and 2 tears are more common in MCL. Osseous/Osteochondral lesions were seen in 17 patients (34\%). Most of these were bony contusions involving the femoral and tibial condyles. INTERPRETATION AND CONCLUSIONS: From these observations, we found that MRI Magnetic resonance imaging is an excellent, non invasive, radiation free imaging modality with multiplanar capabilities and excellent soft tissue delineation. It can accurately detect, localize and characterize various internal derangements of the knee joint and help in arriving at a correct anatomical diagnosis thereby guiding further management of the patient.

KEY WORDS: Traumatic knee; MRI; Arthroscopy; IDK

INTRODUCTION: Trauma to knee joint is a significant cause of morbidity in the young, active individuals especially amongst and athletes. An accurate diagnosis regarding the type and extent of injuries is essential for early operative as well as non-operative treatment. This requires an accurate clinical history, a thorough physical examination and complementary diagnostic tools. With the availability of improved surgical options, accurate imaging of the knee becomes all the more imperative. The most widely used diagnostic modalities to assess the joint injury are arthroscopy 
and MRI. Arthroscopy, though accurate, is invasive and can cause complications ${ }^{1,2}$. Magnetic resonance imaging (MRI) has now been accepted as the best imaging modality for non-invasive evaluation of knee injuries. It has been reported to have a high diagnostic accuracy and does not involve the use of ionizing radiation3. Since its introduction for clinical use in the mid-1980s, the role of MRI in the diagnosis of knee lesions has been well established. MRI has proved reliable, safe and offers advantages over diagnostic arthroscopy, which is currently regarded as the reference standard for the diagnosis of internal derangements of the knee ${ }^{4}$. In the context of trauma, posttraumatic limited range of motion and mechanical knee symptoms MRI is generally considered a valuable diagnostic tool ${ }^{5}$. MRI has made it possible to look into the injured knee non-invasively, thereby avoiding Invasive procedures and further morbidity ${ }^{6}$. The knee joint is a compound type of synovial joint and due to the lack of bony support, stability of the joint is highly dependent on its supporting ligamentous structures, and therefore injuries of ligaments and menisci are extremely common. This study was undertaken to evaluate the types and incidence of injuries in traumatic knee joint by MRI and to compare with arthroscopy / surgical findings in sel ected cases.

MATERIALS AND METHODS: Internal derangements of traumatic knee can be diagnosed by following imaging techniques:

1. Ultrasound

2. Arthrography

3. Computed Tomography

4. Arthroscopy

5. Magnetic Resonance Imaging

1. ULTRASOUND: Sonographical examination has been widely used for detecting musculoskeletal disorders in recent decades. Sonographic examination can accurately detect effusion of the knee. The detection of knee effusion in patients with traumatic knee injury by sonographic examination is highly indicative of internal knee derangement. It has the advantages of being non-invasive, readily available, dynamic, and allows for good visualization of superficial structures. It is well accepted for use in evaluating extra-articular structures of the knee, but is still debated in intra-articular examination. However, the clinical role of sonographical examination in detection of knee effusion is not well explored.

2. ARTHROGRAPHY: It can be used to improve the accuracy of diagnosis .It is invasive and can cause complications. It requires a skilled person for interpreting and reporting the results. It also involves risk of exposure to radiation

3. COMPUTED TOMOGRAPHY: CT provides valuable information about co-existing joint effusion, chondral and osseous fractures and can accurately locate intra articular loose bodies. It also provides a non-invasive method of assessing the cruciate ligaments by thin section CT with reformatting in sagittal plane. This method however is not precise and exposes the patient to ionizing radiations

4. ARTHROSCOPY: Arthroscopy is considered as "the gold standard" for the diagnosis of traumatic intra articular knee lesions, having accuracy as high as $95 \%$ to $98 \%$. However, arthroscopy is an invasive and expensive tool that requires hospitalization and regional or general anaesthesia, thus presenting all the potential complications of an open surgical procedure. Today, knee arthroscopy is 
the most common procedure performed among orthopaedic surgeons. Initially, knee arthroscopy was used as a diagnostic technique. With the advent of magnetic resonance imaging and other noninvasive techniques, knee arthroscopy is now used for the treatment of various knee pathologies. Indications for knee arthroscopy include the treatment of meniscal pathology, specified articular cartilage lesions, osteochondral lesions, loose bodies, advanced synovitis, cruciate ligament tears and certain tibial plateau fractures. One of the most common indications for knee arthroscopy has historically been the debridement of the arthritic knee

5. MAGNETIC RESONANCE IMAGING: MRI has been confirmed as the ideal approach for primary diagnosis of traumatic intra-articular knee lesions. It is non invasive, fast, can be done on an outpatient basis, and is free of complications. It is unique in its ability to demonstrate the nature and extent of injuries. Imaging can be accomplished in any plane without moving the patient and a wide variety of MRI pulse sequences can be performed to produce diagnostic quality images. These include spin echo, fast (turbo) spin-echo, and gradient-echo sequences, which all have been proven suitable for knee imaging. T1 or proton density-weighted sequences are most suitable for visualizing the ligamentous anatomy. Typically, a routine scanning protocol would consist of a combination of one or more of these sequence types performed in the axial, sagittal, and coronal planes using thin sections (maximum $3 \mathrm{~mm}$ with an interslice gap of 0.5 to $1 \mathrm{~mm}$

ANATOMY OF KNEE JOINT: MRI of knee is performed using transmit/receive general purpose extremity surface coil. Quadrature and phase array coils are also available. Increased spatial resolution and decreased signal to noise ratio are significant advantages of these coils. Positioning: Imaging is done with full extension in neutral position. A 14 to $16 \mathrm{~cm}$ field of view and a 3-4 mm slice thickness. MRI is obtained in the Axial, Sagittal and coronal views. Pulse Sequences: A wide variety of MRI pulse sequences can be performed to produce diagnostic quality images. These include spin echo, fast (turbo) spin-echo, and gradient-echo sequences, which all have been proven suitable for knee imaging. T1 or proton density-weighted sequences are most suitable for visualizing the ligamentous anatomy. T2 or STIR sequences with fat saturation are essential to demonstrate bone marrow oedema. Typically, a routine scanning protocol would consist of a combination of one or more of these sequence types performed in the axial, sagittal, and coronal planes using thin sections (maximum $3 \mathrm{~mm}$ with an interslice gap of 0.5 to $1 \mathrm{~mm}$ ). A field-of-view of 12 to $16 \mathrm{~cm}$ depending on patient size is commonly used with a high-resolution matrix of at least 140 steps in the phaseencoding direction and 256 steps in the frequency-encoding direction

Synovial Membrane: It appears on both sagittal and coronal images as linear medium signal intensity on T1 weighted and proton density images and as high signal intensity on T2 weighted images.

Bursae around the knee joint: They are not usually seen on MRI unless they are inflamed and fluid filled.

Anterior Cruciate ligament: ACL is best seen on sagittal, oblique images with slices oriented parallel to the cortex of the lateral femoral condyle. It may appear as a solid band or as three or four separate low signal intensity bands. The tibial attachment is usually better seen than the femoral attachment because of partial volume averaging with lateral femoral condyle. Signal intensity on T1 weighted and spin density images at the tibial insertion is increased. The anterior border of taut ACL should nearly parallel the roof of the intercondylar notch. Coronal and axial images are useful in 
confirming the findings made on sagittal images. Coronal images show the ACL as a curvilinear fan like structure adjacent to the horizontal segment of PCL, near the medial surface of the lateral femoral condyle. Axial images depict ACL as low signal band that is flattened against the medial surface the lateral femoral condyle. All imaging sequences demonstrate fat at the intercondylar notch Posterior Cruciate Ligament: Sagittal images best depict the PCL, which appears as a uniformly low signal intensity structure with a nearly horizontal take off at the femoral origin and then an abrupt descent at about 45 degrees to the tibia (Fig-6). This angled portion of the ligament is normally directed towards the femur. The meniscofemoral ligaments of Humphrey and Wrisburg are seen as low signal intensity dots anterior and posterior to the PCL and should not be mistaken for displaced meniscal fragments or an intact PCL in presence of tear.

Medial and Lateral Collateral Ligament: It is best seen on coronal images where it appears as homogenously low signal intensity structure on all pulse sequences. Moderately increased signal intensities may be seen between and superficial and deep fibres and below the superficial fibres at the distal tibial attachment site, where fat is normally interposed.

Oblique Popliteal Ligament (Posterior Oblique Ligament): It is best seen on coronal and axial images. A coronal oblique plane along the superoinferior course of the OPL (POL) is also optimal for imaging.

The Menisci: The sagittal plane is most important plane in assessing the menisci, with a coronal plane providing supportive rather than new information and the axial plane increasing the accuracy of the sagittal and coronal planes when combined. In general, sagittal images optimally show anterior and posterior horns of the medial and the lateral menisci, coronal images help in evaluating the Meniscal bodies and thin axial sections provide an additional view of menisci and their free edges.

Sagittal image: The anterior and the posterior horns of menisci appear as isosceles triangles. The posterior horn of medial meniscus is twice the size of anterior horn. The anterior and posterior horns of lateral meniscus are of same size. The posterior horn of either menisci should never appear smaller than the anterior horn. On both sides, the menisci appear as flat bands. On lateral side, the more central the slices take on bowtie configuration because of smaller radius of curvature.

Coronal images: Mid portion of the knee produce best images of bodies of both menisci. They appear triangular and slightly larger laterally than medially. The capsular attachment on the medial side is incorporated in the tibial and medial collateral ligament. A small amount of fat may be interposed between the body of the medial meniscus and the capsule. On posterior coronal cross sections, the posterior horns appear as flat bands. On lateral cross sections, the popliteal tendon courses upward and laterally at 45 degrees. More anteriorly, the anterior horn of lateral meniscus appears as a band like structure. The anterior horn of medial meniscus extends more anteriorly than that of lateral meniscus. The medial and lateral menisci, the transverse ligament, and the meniscofemoral ligament appear homogenously dark on all pulse sequences. The vascular and avascular zones cannot be distinguished on MRI. The vascularized zone does not demonstrate enhancement with intravenous gadolinium.

\section{PATHOGENESIS AND MAGNETIC RESONANCE IMAGING APPEARANCES OF INTERNAL DERANGEMENTS OF KNEE}


The traumatic internal derangements of the knee can be classified as those involving,

i. Cruciate ligaments

ii. Collateral ligaments

iii. Menisci

iv. Extensor mechanism and the patellar tendon

v. Osseous and osteochondral injuries

\section{i) THE CRUCIATE LIGAMENTS}

Anterior Cruciate Ligament: Most common mechanism of injury to anterior cruciate ligament is external rotation of femur on a fixed tibia with a valgus force. Other less common mechanisms include hyperextension, internal rotation with maximal extension or anterior translation of tibia produced by a direct force against the back of calf. Clinically the patients present with hemarthrosis and an audible pop at the time of injury. The anterior cruciate ligament has limited healing capacity possibly related to heterogeneity of collagen fibres or fibroblast function within a synovial environment. Acute rupture of the ACL ranges from low grade, partial thickness to full thickness and are located most commonly in the mid- to proximal aspect of the ligament. ACL tears occur up to eight times more commonly in females than in males. A torn ACL fibre has increased T2- weighted signal and an abnormal contour. In some full-thickness tears, an amorphous mass replaces the discrete ACL fibres. Fluid can fill the gap between the fibres of a full-thickness tear. The location of the tear can be described as proximal, midsubstance, distal, or involving the femoral or tibial attachment.

Women are more likely to have tears involving the proximal ACL, likely because of the higher incidence of non-contact inciting mechanisms. Avulsion of the ACL from the tibial attachment site is more common in young patients.

On MR imaging there are both direct and indirect signs of anterior cruciate ligament injury.

\section{The direct signs include:}

i) Discontinuity of the fibres of anterior cruciate ligament

ii) An abnormal contour of anterior cruciate ligament.

\section{The indirect signs include:}

i. Angulation of the posterior cruciate ligament of less than $105^{\circ}$

ii. Anterior tibial translation exceeding $6 \mathrm{~mm}$

iii. Overhanging posterior horn of the lateral meniscus by $2.5 \mathrm{~mm}$

iv. Deep lateral femoral notch exceeding $2 \mathrm{~mm}$ in depth

v. Segond fracture of the lateral tibia involving the middle portion of the lateral fibrous capsule at the meniscofemoral attachment

vi. Chip fracture of the posterior tibia

vii. Rotary bone contusion pattern.

viii.Irregularity of the free concave edge of Hoffa's fat pad suggestive of synovitis

The site of an ACL tear is easier to identify during the sub-acute phase. The "double PCL" sign classically is seen during this phase with the distal end of the torn ACL anterior and parallel to the 
PCL [34]. Occasionally, the proximal end of the ACL may be displaced posterior to the PCL, simulating a large, loose body. Chronic ACL tears are seen in several different ways on MRI. If the ligament has completely atrophied, it will not be visualized. There may be a small attachment on the tibia left in chronic tears. The distal end of the ACL may also be intact and attached to the PCL without visualization of the proximal end. This is commonly mistaken for an intact ligament on MRI.

\section{Posterior Cruciate Ligament:}

The PCL is injured by one of three mechanisms:

(1) Knee hyperextension,

(2) Knee hyper flexion, or

(3) Posterior displacement of the tibia with the knee flexed.

The PCL most commonly tears at the mid portion of the ligament, and is more likely than the ACL to have a partial tear. PCL injuries have a high association with other injuries. Trabecular micro fractures, or bone bruises are seen associated commonly. PCL tears are also associated with posterolateral corner injuries. Acute tears of the PCL are usually manifested by thickening of the middle portion of the ligament with increased signal on both T1- and T2- weighted images. The mass like quality that an acutely torn ACL exhibits is not seen in acute PCL tears. Also, unlike acute ACL tears, continuity of the ligament is maintained in the PCL with acute trauma.Chronic tears of the PCL are difficult to diagnose and image. Because most PCL injuries heal without surgery, the ligament will form fibrous scar in the place of normal ligament. This scar generally has the same signal intensity of native tissue. Secondary signs such as a lax ligament or persistent increased signal may help diagno se chronic PCL tears.

\section{ii) THE COLLATERAL LIGAMENTS}

Medial Collateral Ligament (MCL): MCL injuries are usually caused by valgus stress, and are often associated with other knee derangements. The combination of an ACL tear, MCL tear, and medial meniscal tear occurs with valgus stress to the knee while the foot is fixed on the ground, and is also known as the "unhappy triad" or O' Donoghue's triad. The traditional classification of MCL tears is to grade the damage as first- through third-d egree sprain/tear injuries.

Grade I: Lesions are defined as high signal intensity superficial to the MCL representing oedema, with intact MCL fibres.

Grade II: Lesions in which fluid signal extend partially through the MCL, although some fibres remain intact

Grade III: Lesions with complete discontinuity of the MCL fibres seen along with surrounding oedema, consistent with a complete rupture.

Lateral Collateral Ligament (LCL): Almost all tears of the LCL are associated with other injuries. These injuries include damage to structures within the region of the LCL, such as the capsule, the biceps femoris, and the popliteus, as well as to structures elsewhere in the knee, such as the cruciate ligaments or the lateral tibial rim (Segond fracture) Like the MCL, the LCL is best visualized on coronal images. Lateral collateral ligament injuries are usually classified similarly as medial collateral ligament lesions, using the 3-point grading system as outlined above. Lesions of the LCL 
are most often caused by varus stress and therefore may be associated with impression fractures of the medial tibial plateau. In addition, LCL ruptures are frequently seen together with other injuries of the posterolateral corner.

iii) THE MENISCI: Abnormal shearing forces may be generated during compression and rotation of knee resulting in meniscal damage. The rotation of femur on a fixed tibia during flexion and extension places the menisci at risk for injury. Clinical signs of meniscal injury include joint pain along the joint line, giving way, clicking, locking of knee in fixed flexion and effusions. Before embarking upon the pathological states, it is worthwhile considering normal anatomic variants which should not be mistaken for pathological conditions. Before embarking upon the pathological states, it is worthwhile considering normal anatomical variants, which should not be mistaken for pathological conditions.

NORMAL MENISCAL VARIANTS: The Discoid Meniscus: It is a congenital anomaly found usually in lateral meniscus. It has reported incidence of 0.4 to $16.6 \%$. A meniscus is said to be discoid if there is continuity between anterior and posterior side [bow tie appearance] on five or more contiguous $3 \mathrm{~mm}$ sagittal MRI section. It presents with symptoms of joint line tenderness, "snapping", and locking of the knee. The three types of discoid lateral meniscus are complete, incomplete, and the Wrisburg variant. Some investigators include a ring-shaped meniscus's a fourth type. The complete and incomplete types have a firm, normal posterior tibial attachment and are stable. Symptomatic patients who have these types of discoid menisci usually are treated with a partial meniscectomy. In contrast, the Wrisburg variant has no posterior coronary or capsular attachments and increased T2 signal is present between the meniscus and the capsule simulating a peripheral tear or a fascicular, injury. The Wrisburg variant has the most notable symptoms, with a "snapping' sensation occurring when the posterior horn moves across the femoral condyle during flexion and extension. A discoid medial meniscus is much less common, with the incidence reported to be $0.12 \%$ to $0.6 \%$. On MR, the diagnosis of a discoid meniscus is suggested by identifying either meniscal tissue on three continuous sagittal 5 -mm-thick slices, or a meniscal body on coronal images greater than $15 \mathrm{~mm}$ wide or extending into the intercondylar notch. The discoid meniscus has an increased incidence of tears and degeneration, likely caused by its abnormal shape, resulting in increased stress on the meniscus. Intrasubstance "grade 2" signal, or abnormal signal not extending to an articular surface, is noted in $24 \%$ of discoid menisci and is more common in complete discoid menisci. Typically, this abnormal signal is not considered clinically significant. However, in the population with discoid menisci, some investigators report that this Intrasubstance signal may be significant clinically.

\section{GRE coronal image of discoid meniscus}

Meniscal Ossicles: They are reported in $0.15 \%$ of patients and are thought to be either developmental or posttraumatic. These small, ossific foci are found typically in the posterior horn of the medial meniscus and are associated with meniscal tears. They can be asymptomatic, or associated with pain and a sensation of locking, clinically simulating a torn meniscus with a flap component. The ossicle follows the signal of bone marrow on MRI .Meniscal flounce: It is a wavy appearance along the free edge of the meniscus. Previously, meniscal flounce was thought to be identified only at arthroscopy, in the presence of joint fluid in the setting of an ACL or medial 
collateral ligament (MCL) tear. However, a flounce can be seen without a ligament injury. Recently, the Meniscal flounce has been identified with MR imaging when the knee is in 10 degrees of flexion.. The flounce can appear truncated on coronal images and can simulate a tear or degeneration. The incidence at MR is reported to be from $0.2 \%$ to $6 \%$. A flounce-like appearance can be seen with meniscal tears. Meniscal Extrusion: It is measured from the outer meniscal edge to the proximal tibial margin. Extrusion of the medial meniscus more than $3 \mathrm{~mm}$ is considered abnormal. This degree of extrusion can be seen in patients who have advanced Meniscal degeneration, and various types of meniscal tears. Although extrusion of the anterior horn or body of the lateral meniscus sometimes is considered a normal variant, others consider extrusion of the lateral meniscus more than $1 \mathrm{~mm}$ to be abnormal.

\section{MENISCAL TEARS:}

The cause of meniscal tears can be divided into two categories.

a) Increased force on a normal meniscus, usually resulting in longitudinal or radial tears, and

b) Normal forces on a degenerative meniscus, usually producing horizontal tears in the posterior half of the meniscus.

Tears are more common in the medial meniscus, possibly because the medial meniscus is less mobile, and it bears more force during weight bearing than the lateral meniscus, with $56 \%$ of tears involving the posterior horn of the medial meniscus. Tears isolated to the anterior two thirds of the meniscus are uncommon, representing only $2 \%$ of medial and $16 \%$ of lateral meniscal tears .Lateral meniscal tears are more common in younger patients (under 30 years old), who have a higher incidence of tears related to sporting events than do older patients. It is likely that this is related to the higher incidence of concomitant ACL tears in this population.

Diagnostic Criteria: Two criteria for diagnosing a meniscal tear are commonly used:

(i) An Intrasubstance area of intermediate or high signal intensity that unequivocally extends to the articular surface,

(ii) Abnormal meniscal morphology.

i) Intrasubstance signal: It is graded as follows:

Grade 1: Intra meniscal high signal intensity of irregular or globular appearance that is confined within the meniscus and does not extend to the articular surface.

Grade 2: The signal is linear and does not intersect the inferior or superior articular surface. It may, however, contact the capsular margin at the posterior aspect of the meniscus.

Grade 3: Tears characterized by linear high or intermediate signal intensity that extends to the superior and/or inferior articular surface.

Grade 4: It is sometimes added to indicate a complex tear with multiple components or fragmentation.

Both grade 1 and grade 2 lesions do not represent a tear, but indicate mucinous and mucoid Intrasubstance degenerative change and are usually encountered after the third or fourth decade. In children and adolescents, prominent vascularity may resemble grade 1 or grade 2 lesions. 
ii) Abnormal Meniscal morphology: Morphologic changes of the meniscus associated with meniscal tears include blunting of tip of the inner free meniscal edges of the meniscus, displacement of a portion of the meniscus, interrupted appearance of the meniscus and abnormal size of a segment of the meniscus is detached (a bucket handle tear), the remaining peripheral portion appears small and often truncated. The displaced portion usually lies within the intercondylar notch beneath the PCL. Coronal views are helpful in further identifying such displaced fragments. An abrupt change in contour of the meniscus, known as the 'notch' sign, is an important indicator of a meniscal tear. Although the normal meniscal flounce can simulate it, the presence of abnormal intra meniscal signal makes the notch sign a more definite indicator of a meniscal tear.

\section{Classification of tears:}

1. Horizontal tears

2. Vertical tears

3. Complex tears

1. Horizontal Tears: Horizontal tears are parallel to the tibial plateau and divide the meniscus into upper and lower segments. The Cleavage type extends to the apex and divides the menisci into upper and lower halves. They are referred to as 'fish mouth' tears. In the Non cleavage type, tear extends to the superior and inferior articular surface of the meniscus. Horizontal tears are most common within the posterior horn of the medial meniscus. Linear horizontally oriented grade II signal intensity is sometimes mistaken for complete horizontal tears .Sagittal GRE image demonstrating a horizontal tear of the posterior horn of the medial meniscus.

2. Vertical Tears: They are perpendicular to the tibial plateau. A vertical longitudinal tear occurs between the circumferential collagen fibres parallel to the long axis of the meniscus. Longitudinal tears can be obliquely oriented, but are not parallel to the tibial plateau. Vertical grade III signal intensity located within the peripheral third of the meniscus is considered a peripheral longitudinal tear. A vertical radial tear occurs perpendicular to the circumferential collagen fibres and along long axis of the meniscus. Radial tears are also known as free edge tears because they involve inner edge of the meniscus. MR images obtained perpendicular to the orientation of radial tear easily demonstrates tear as a small gap or cleft in the meniscus (Cleft sign), which sometimes can be observed to move across the meniscus on successive images (Margin Cleft sign). If the orientation of the imaging plane is in the same direction as a full thickness radial tear, a section through the same plane of the tear will demonstrate the absent meniscus or high signal intensity not representative of meniscus because of volume averaging, which results in the so called Ghost sign. A partial thickness radial tear will demonstrate blunting or abrupt truncation of the apex of the meniscus (truncated triangle sign). The term parrot beak tear has been frequently used to describe a vertical radial tear at the inner edge of the meniscus that curves obliquely across the circumferential collagen fibres. Root tears: They are full thickness radial tears at the central tibial attachment sites of the menisci \& are called the root ligaments. .A root tear is reportedly difficult tear to diagnose because meniscal tissue is noted only on one side of the tear. The diagnosis is easier to make medially because of the close anatomic relationship between posterior horn of the meniscus and the tibial attachment of the PCL. Normally, on 3-mm sagittal images, the meniscus should be seen on the image medial to the 
PCL attachment; otherwise, a root tear is suspected and the coronal images can confirm. Lateral meniscal root tears are diagnosed when posterior horn of the lateral meniscus does not cover the most medial aspect of posterior lateral tibial plateau on at least one coronal image.

Bucket-handle tears: These are displaced longitudinal tears. The displaced in a fragment often has the appearance of a handle, and the remaining peripheral segment attached to the tibial resembles a bucket - hence the name. Confirmation of this tear is mandatory and can be made by identification of displaced fragment. The most reliable sign is the finding of a displaced fragment of meniscus in the intercondylar notch, which can be better seen on coronal images. This sign is associated with a truncated or shortened meniscus on coronal images. On sagittal images, the displaced fragment can be seen lying anterior and parallel to the PCL. This is known as the double PCL sign. These tears may also result in the flipped meniscus sign, double delta sign, or double anterior horn sign, in which the displaced meniscal segment of the posterior horn is located anterior to, on top of, or posterior to the anterior horn. Helpful clues in these cases include non-visualization of the posterior horn of the meniscus, the appearance of a larger anterior horn than posterior horn, or an anterior horn taller than $6 \mathrm{~mm}$. Flap Tears: A flap tear or a displaced flap tear is a term that is used often to describe a short-segment, horizontal meniscal tear with fragments either displaced into the notch or into the superior or inferior gutters. Flap tears are the most common type of meniscal tears and may develop following only minimal meniscal trauma of the degenerative meniscus from chronic shear stress. They involve the inner one third to one half of the meniscus with a superior or inferior leaf extension that creates the flap.

3. Complex tears: They either have two or more tear configurations or are not categorized easily into a certain type of tear.

MRI grading of meniscal tears and degenerations: A grading system based on the signal abnormality correlated with histopathological abnormalities has been developed to understand the significance of increased signal intensity patterns in meniscal abnormalities.

Grade I: Non-articular focal or globular intrasubstance increased signal intensity in the non articular portion of the meniscus.

Grade II: Horizontal linear intrasubstance increased signal intensity usually extend s from the capsular periphery but does not involve an articular surface.

Grade III: Region of abnormal signal intensity extends or communicates to at least one of the articular surfaces.

MENISCAL CYST: A meniscal cyst is a fluid-filled structure adjacent to a meniscus, which appears as Intermediate signal intensity mass on T1-weighted images and hyper intense on T2-weighted images, sometimes lobulated or septated. Meniscal cysts are strongly associated with a horizontal cleavage tear of the meniscus and often a connecting neck between the tear and the cyst can be visualized. The cysts may become fairly large, which is thought to be caused by a ball valve mechanism. They can become symptomatic usually on the medial side, since they may impress on the medial collateral ligament.

MENISOCAPSULAR SEPERATION: Menisco-capsular separation occurs when the meniscus detaches from the capsular attachments, which is more common medially and usually is associated 
with other injuries. Menisco-capsular separation is evaluated best on coronal and sagittal T1- or proton density- weighted sequences for anatomy, and fat-saturated T2-weighted or STIR sequences for pathology. Signs that have been described in menisco-capsular separation include displacement of the meniscus relative to the tibial margin, extension of the tear into the superior or inferior corner of the peripheral meniscus, and an irregular outer margin of the meniscus body on coronal images. Additional signs include increased distance between the meniscus and the MCL, or fluid between the meniscus and the MCL. Overall, the presence of perimeniscal fluid and an irregular meniscal outline are the best predictors of meniscocapsular separation.

IV) EXTENSOR MECHANISM AND PATELLAR TENDON: The extensor mechanism of the knee consists of the quadriceps muscle group, quadriceps tendon, patella, patellar retinaculum, patellar ligament, and adjacent soft tissues. Injuries to the extensor mechanism are common and consist of chronic degenerative injuries, overuse injuries, and acute trauma. Overuse injuries typically are seen in high performance athletes, most commonly in sports requiring long distance running or jumping. Cumulative micro trauma produced by repetitive knee flexion and extension results in focal inflammation .Acute traumatic injuries occur secondary to direct blunt trauma or excessive tension applied to the extensor mechanism through quadriceps contraction. Patellar fractures are the most common traumatic injury of the knee extensor mechanism.

Patellar tendinopathy: Patellar injuries can result from chronic overuse injuries or as a result of acute trauma. Patellar tendinosis or 'jumper's knee' results from chronic overload on the patellar tendon because of strain from the quadriceps muscle group. Chronic overload results in micro tears and focal degeneration, which is characterized histologically by pseudocyst formation, increased fibrocartilage, and myxomatous and hyaline metaplasia. Patellar tendinosis frequently is seen in participants in sports associated with jumping and is associated with mal-alignment. MRI findings consist of focal thickening of the proximal patellar tendon, with increased signal on T1-weighted and T2-weighted images. Oedema in the paratenon, infrapatellar fat pad, and subcutaneous adipose tissue is associated with acute patellar tendonitis, which is defined as symptoms of less than 2-week duration and patellar tendon tears. Acute patellar tendonitis is associated with increased intrasubstance signal on T1-weighted and T2-weighted images without tendon thickening.

Patellar tendon tears: They may be partial or complete, and most frequently occur in the proximal inferior patellar tendon. A patellar sleeve fracture represents a cartilaginous avulsion from the lower pole of the patella and is often associated with patellar tendon tears. With complete inferior patellar tendon tears, the patella retracts proximally as a result of the pull of the superior patellar tendon. MRI shows discontinuity of the tendon, demonstrated on fluid-sensitive sagittal T2-weighted and STIR images. MRI findings in partial patellar tendon tears can resemble acute patellar tendonitis. Correlation with a history of acute trauma is useful for specific diagnosis.

Quadriceps tendon tear: A tear of the quadriceps tendon is the second most common acute injury to the extensor mechanism. Injury to the quadriceps mechanism (rectus femoris, vastus medialis, vastus intermedius, vastus lateralis, and quadriceps tendon) may be the result of acute trauma caused by rapid deceleration, such as running when the foot is planted, or it may be caused by 
chronic micro trauma. In elderly patients or patients who have gout, diabetes, connective tissue diseases, or other systemic conditions, minor trauma may result in quadriceps injury MRI is useful in differentiating complete from partial quadriceps tendon tears. With complete tears, discontinuity of the tendon with associated retraction of the proximal remnant usually is visualized best using T2weighted images. Often, an associated hematoma is seen with heterogeneous signal intensity on T1weighted images.

Myotendinous strain: Myotendinous strain is a common injury in athletes. The most commonly injured muscles are fusiform muscles that cross two joints. In these muscles, high tensile stress develops when the muscle is forced to lengthen during active contraction, a process termed eccentric contraction. Because the rectus femoris muscle is the only component of the quadriceps muscle group that crosses two joints, it is the most common site for myotendinous strain injuries in the knee extensor mechanism .MRI findings of strain injuries consist of focal edema centered on the central tendon of the rectus femoris. The presence of a focal hematoma is indicative of a partial myotendinous tear. Complete myotendinous tears often are recognized clinically, and these patients less frequently undergo MRI.

V) OSSEOUS AND OSTEOCHONDRAL LESIONS: Bone bruise or marrow edema pattern: Bone bruises or marrow edema may be identified with numerous conditions, including trauma, infection, and osteoporosis. Bone bruises related to trauma may be caused by a direct blow, articular compression forces, or avulsion injuries. The extent of marrow edema tends to be more dramatic with compression or direct trauma, compared with avulsion injuries. Typically, bone bruises are not visible on radiographs. However, these injuries are detected easily on MR images using T1-weighted sequences and either short T1 inversion recovery (STIR) or fat-suppressed T2-weighted sequences. Specific edema patterns are also useful in predicting the mechanism of injury and the associated ligament, tendon, or meniscal involvement.

Tibial plateau fractures: Subtle tibial plateau fractures are overlooked easily on radiographs. The only finding may be lipohemarthrosis, which can be detected on the cross table lateral radiograph. Displaced tibial plateau fractures are detected easily on radiographs. However, further imaging with CT or MR imaging is required to evaluate fragment position and associated soft-tissue injuries. CT is adequate for the evaluation of fracture position, articular step-off, and separation of fragments. MR imaging, especially with subtle fractures, can assess the bony injury and associated ligament and meniscal injury more easily.

Segond and reverse Segond fractures: The Segond fracture is a small, avulsion fracture proximal and posterior to the insertion of the iliotibial band on Gerdy's tubercle. The avulsion may occur with internal rotation and the knee flexed, or with internal rotation and varus stress. The fracture is caused by avulsion of the meniscotibial portion of the middle third of the lateral capsular ligament, with possible associated fractures of the fibular head or Gerdy's tubercle. In the case of sever e varus stress, such as in a motor vehicle accident, the entire lateral ligament complex may be disrupted. Segond fractures have a high incidence of anterior cruciate ligament tears and meniscal injury. MR imaging is ideal for the detection of osseous, meniscal, and ligament injuries. The reverse Segond 
fracture, much less common, is a medial avulsion of the deep medial collateral ligament attachment on the tibia. The mechanism of injury is felt to be valgus stress with external rotation and is associated with disruption of the posterior cruciate ligament and peripheral medial meniscus tears. When this avulsion is detected on radiographs, MR imaging should be performed to evaluate the associated ligament and meniscal injuries.

Osteochondral lesions: Osteochondral lesions in the knee may be acute or chronic. Acute lesions result from impaction, or rotational or shearing forces. The fracture may involve only cartilage, or cartilage and underlying bone. The fracture line is parallel to the joint line, unlike more conventional fractures that enter the joint vertically or obliquely. Fractures may be impacted, el evated partially or displaced completely and free in the joint. When imaging osteochondral lesions, radiographs may be useful, but detection and appropriate classification are accomplished more effectively with MR imaging using T1- and T2-weighted or STIR sequences. The axial and sagittal planes are most useful for femoral lesions. Lesions that are minimal (softening, fibrillation, or fissuring) show abnormal signal intensity without elevation or separation. MR signs of unstable lesions include a high signal intensity line between the native bone and the osteochondral fragment, focal defects, articular fracture, and adjacent subchondral cysts.

Spontaneous osteonecrosis of the knee: Spontaneous osteonecrosis of the knee (SONK): Spontaneous osteonecrosis of the knee was considered classically a condition of vascular insufficiency, leading to bone infarction of the weight bearing surface of the femoral condyle. The condition was seen in older adults. The condition almost always was unilateral and involved most commonly the medial femoral condyle. Growing base of knowledge in the literature suggests that a subchondral insufficiency fracture leads to focal osteonecrosis. Similar features have been described in the medial tibial plateau as well

METHODOLOGY: 50 cases with history of trauma to the knee, who were referred to radiology department from orthopaedic department of NMCH \& RC, Raichur, suspecting internal derangement of knee, served as the subjects for this study.

\section{MATERIALS AND METHODS:}

1. Source of Data: All cases referred from orthopaedic department of NMCH \& RC for evaluation of traumatic knee.

2. Method of data collection
a) Duration of study : $2011-2013$.
b) Sample size $\quad: 50$ cases.
c) Inclusion Criteria : All the patients referred with clinically suspected internal derangement of knee following trauma to knee
d) Exclusion Criteria: i) Patients with ferromagnetic implants, pacemakers, and aneurysm clips.
ii) Patients with major injuries like liver / splenic rupture and flail chest and patients with unstable vital parameters especially in the setting of trauma


e) Methods

: All patients will be subjected to MR imaging and followed by arthroscopy / surgery in selected cases wherever indicated.

3. Imaging protocols

Patients will be subjected to MRI within 4 days of clinical evaluation according to the following protocols:

Equipment: Siemens Magnetom Avanto 1.5 Tesla. Whole- body MR scanner

Protocol: i. T1 \& PD weighted sequences in sagittal and coronal planes.

ii. T2- weighted in axial, coronal and sagittal planes.

iii. Fat suppressed T2 or STIR sequences wherever indicated.

4. Interpretation of MRI data:

Study will be evaluated for:

Joint effusion: Present/Absent

Anterior Cruciate Ligament tear: Present/Absent

Posterior Cruciate Ligament tear: Present/Absent,

Partial/Complete

Medial Collateral Ligament tear: Present/Absent

Grade- 1/2/3

Lateral Collateral Ligament tear: Present/Absent

Grade- 1/2/3

Medial Meniscal Tear: Present/Absent

Location \& Type

Lateral Meniscal Tear: Present/Absent

Location \& Type

Osseous /Osteochondral lesions: Present/Absent

Location \& Type

5. Arthroscopy/ Surgery: Will be carried out in OT by orthopaedic surgeon in selected cases where indicated for diagnostic or therapeutic purposes.

6. Research hypothesis: Findings of MRI of the Knee are well correlated with the arthroscopic findings in patients of trauma to the Knee.

Study Design: A descriptive statistical analysis and correlation evaluation study consisting of 50 patients with Traumatic knee joint is undertaken to study the spectrum of MRI findings in all consecutive cases of knee trauma referred from orthopaedic OPD of NMCH \& RC and comparison of findings of magnetic resonance imaging with arthroscopic findings in selected cases. Sensitivity, specificity, PPV, NPV and Accuracy could not be found as the Arthroscopic/Surgical intervention required only after the positive indication of MRI diagnosis. Therefore, the both the variables are not statistically independent hence the diagnostic statistics cannot be performed.

RESULTS: Study design: A descriptive statistical analysis and correlation evaluation study consisting of 111 patients with traumatic knee joint is undertaken to study the spectrum of MRI findings in all consecutive cases of knee trauma referred from orthopedic OPD of CHAF and comparison of findings of magnetic resonance imaging with arthro scopic findings in selected cases. 
In our study joint effusions were the most common finding affecting 37 patients (74\%). Among the ligamentous and meniscal injuries, ACL tear is most common, seen in 22 patients (44\%), to be followed by the MCL and Medial Meniscal injuries seen in 19 patients (38\%). In our study ACL tear was found in 22 patients (44\%) among these 7 (31\%) were partial tears and $15(68 \%)$ were complete tears. Posterior cruciate ligament injuries were found to be relatively uncommon, in our study found in only 4 patients (8\%) and all the tears were demonstrated as thickening of the ligament with abnormal signal intensity (partial tear). In our study, MCL tears (38\%) were found to be more common than the LCL tears (8\%).

In our study, MM tears were found in 19 (38\%) with Grade I tear in 6 (31.5), Grade 2 tear in 10 (52.6\%) and Grade 3 in 3 (15.7) and LM tears in 9 (18\%) with Grade I tear in 3 (33.3\%), Grade 2 tear in $6(66.6 \%)$ and Grade 3 in none. Grade II tears were the more common in both the menisci; MM 10 (52.6\%) and LM 6 (66.6\%).Lateral Discoid meniscus with appearance of bow tie configuration in more than two contiguous slices on sagittal images was found in one patient.

In our study Osseous/Osteochondral lesions were seen in 17 patients (34\%). Most of these were bony contusions involving the femoral and tibial condyles. Osteochondral lesions are seen in nine patients. In our study we found a case of Comminuted bicondylar fracture of proximal end of Tibia with intra-articular and intracondylar extension. There is also fracture of medial tibial condyle in postero-lateral aspect which is displaced antero-medially. A case of Segond fracture of the lateral tibial rim with bony contusion of the lateral femoral condyle was also seen. The finding of hemarthrosis and lipohemarthrosis was associated in two cases with presence of intercondylar fractures. The findings of a partial tear of proximal fibers of medial head of Gastrocnemius muscle were seen in two patients in our study.

In our study, a correlation of MRI findings with arthroscopic/surgical findings was performed in 20 patients (40\%). Among which in 17 patients (85\%) MRI findings are well correlated with arthroscopic/surgical findings. In our study, we found 29 cases of combined injuries and 21 cases of isolated injuries. The predominant pattern is ACL tear and MM tears; followed by ACL tear and LM tear, in a series of 17 cases of concomitant injuries at MRI and arthroscopy. The predominant pattern was anterior cruciate ligament rupture and medial meniscus tear (5 patients), followed by anterior cruciate ligament and lateral meniscus (4 patients), or anterior cruciate ligament + medial meniscus + lateral ligament (4 patients). In our study we found a case of Comminuted bicondylar fracture of proximal end of tibia with intra-articular and intracondylar extension and multiple ligamentous and Meniscal injuries. The present study revealed the ability of magnetic resonance imaging in evaluation of the various internal derangements, including their detection, localization, characterization and assessment of extent of damage and the strength of correlation between MRI and arthroscopic findings confirms the value of MRI in assessing internal knee structures.

DISCUSSION: The role of magnetic resonance imaging has steadily increased and now it has become the first line investigation for most of the lesions of knee. It is also being used for pre and postoperative evaluation. Complete evaluation of all the internal structures of the knee was not possible with other modalities like conventional radiography, arthrography, ultrasonography and computed tomography. Even with arthroscopy lesions such as peripheral meniscal tears, inferior surface tears and osteochondritis desiccans without articular cartilage damage are most often not detected. 
Multiplanar MR images provide significant improvement in assessing these structures. In our study joint effusions were the most common finding affecting 37 patients (74\%). Among the ligamentous and meniscal injuries, ACL tear is most common, seen in 22 patients (44\%), to be followed by the MCL and Medial Meniscal injuries seen in 19 patients (38\%). Cruciate ligaments: Singh JP et al in their series of 173 patients, 78 patients (45.08\%) showed ACL tears, among these 52 (66.67\%), are partial, $16(20.51 \%)$ are complete and $10(12.82 \%)$ cases showed non visualization of ACL. The authors concluded that ACL tears are more common than other ligamentous injuries with partial tears being commoner. However in our study ACL tear was found in 22 patients (44\%) among these 7 (31\%) were partial tears and 15 (68\%) were complete tears. Posterior cruciate ligament injuries were found to be relatively uncommon, in our study found in only 4 patients (8\%) and all the tears were demonstrated as thickening of the ligament with abnormal signal intensity (partial tear). Sonnin et al found the incidence of PCL tear to be 3 percent; in a series of study analysing 350 case of knee injury only 10 patients had PCL tear. In a study by Grover et al where they analysed findings of 510 consecutive MRI of knee joints with an emphasis on PCL tear; 11 (2\%) patients had different grades of tear on MRI which was confirmed correctly by arthroscopy. Of the other 202 patients who had undergone MRI as well as arthroscopy for internal derangement of knee none of the patients had any PCL injury as predicted correctly by MRI.

Collateral ligaments: In our study, MCL tears (38\%) were found to be more common than the LCL tears (8\%). All these cases had history of trauma and were associated with multiple injuries. This suggests presence of a single injury should prompt the examiner to look for other subtle associated injuries, which was further confirmed by Mink JH et al. They observed on MRI and arthroscopy of 11 patients who had tear of ACL, 7 patients had tear of MCL, 4 patients had tear of lateral meniscus and 1 patient had tear of medial meniscus.

Menisci lesions: There is preponderance of MM tears over LM tears in our study which is well correlated with the study done by Singh JP et al, in a series of 173 cases of which they found 57 (38.23\%) patients showed MM tear and 28(29.41\%) patients showed LM tear. Out of 173 patients, Grade 3 tear of MM was seen in $57(32.95 \%)$ patients, Grade 2 in $16(9.25 \%)$ patients \& Grade 1 in $20(11.56 \%)$. In LM, Grade 3 tears were seen in 28(16.18\%) patients, Gr 2 in 12 (6.94\%) patients \& Gr I in 14(8.1\%) patients[6].In our study, MM tears were found in 19 (38\%) with Grade I tear in 6 (31.5), Grade 2 tear in 10 (52.6\%) and Grade 3 in 3 (15.7) and LM tears in 9 (18\%) with Grade I tear in $3(33.3 \%)$, Grade 2 tear in $6(66.6 \%)$ and Grade 3 in none. Grade II tears were the more common in both the menisci; MM 10 (52.6\%) and LM 6 (66.6\%).Lateral Discoid meniscus with appearance of bow tie configuration in more than two contiguous slices on sagittal images was found in one patient. The cystic lesions encountered were meniscal cyst, parameniscal cyst and popliteal cyst (Baker's cyst). The meniscal cysts and parameniscal cysts were found to be associated with tear of the lateral meniscus in 6 and medial meniscus in 3. In the 2 cases of the popliteal cysts, its location, relation to the joint space and its communication with joint space ware clearly demonstrated on sagittal T2 weighted images. These findings were correlated with findings described by Thomas $\mathrm{H}$. Berquist ${ }^{7}$ 
Osseous and Osteochondral injuries: In our study Osseous/Osteochondral lesions were seen in 17 patients (34\%). Most of these were bony contusions involving the femoral and tibial condyles. Osteochondral lesions are seen in nine patients. In our study we found a case of Comminuted bicondylar fracture of proximal end of Tibia with intra-articular and intracondylar extension. There is also fracture of medial tibial condyle in postero-lateral aspect which is displaced antero-medially. A case of Segond fracture of the lateral tibial rim with bony contusion of the lateral femoral condyle was also seen. These findings were correlated with findings described by Thomas H. Berquist7 ${ }^{7}$. In our study we also saw a case spontaneous osteonecrosis of medial femoral condyle, which is well depicted on magnetic resonance imaging earlier than radiographic appearance. It appeared as a focus of altered signal in the antero-medial aspect of medial femoral condyle with a thin rim of sclerosis and associated marrow edema involving entire medial femoral condyle and flattening of the articular surface with evidence of subchondral collapse. These findings were correlated with findings described by Thomas H. Berquist 7 the finding of hemarthrosis and lipohemarthrosis was associated in two cases with presence of intercondylar fractures. These findings were correlated with findings described by Thomas H.Berquist ${ }^{7}$.The findings of a partial tear of proximal fibres of medial head of Gastrocnemius muscle were seen in two patients in our study. These findings were correlated with findings described by Thomas H.Berquist 7 In our study, a correlation of MRI findings with arthroscopic/surgical findings was performed in 20 patients (40\%). Among which in 17 patients (85\%) MRI findings are well correlated with arthroscopic/surgical findings.

Combined injuries: In our study, we found 29 cases of combined injuries and 21 cases of isolated injuries. The predominant pattern is ACL tear and MM tears; followed by ACL tear and LM tear, which is well correlated with a study by Ali Akbar Esmaili Jah et al, in a series of 17 cases of concomitant injuries at MRI and arthroscopy. The predominant pattern was anterior cruciate ligament rupture and medial meniscus tear ( 5 patients), followed by anterior cruciate ligament and lateral meniscus ( 4 patients), or anterior cruciate ligament + medial meniscus + lateral ligament (4 patients). In our study we found a case of Comminuted bicondylar fracture of proximal end of tibia with intra-articular and intracondylar extension and multiple ligamentous and Meniscal injuries. The present study revealed the ability of magnetic resonance imaging in evaluation of the various internal derangements, including their detection, localization, characterization and assessment of extent of damage and the strength of correlation between MRI and arthroscopic findings confirms the value of MRI in assessing internal knee structures.

SUMMARY \& CONCLUSIONS: The present study attempts to determine the role of magnetic resonance imaging in the evaluation of internal derangements of the knee joint. Knee joint being the largest and most complex weight bearing joint of the body is subject to damage because of its inherent structural complexity and the various types forces it is subjected to Magnetic resonance imaging has emerged as the frontline investigation for evaluation of Internal derangements of the knee joint. It is non invasive, does not involve ionizing radiation and has multiplanar capability with excellent soft tissue demonstration. Arthroscopy gives excellent visualization of the interior of the joint, but is invasive and can evaluate only the surface abnormalities. In the present study, 111 patients with knee joint symptoms, referred for magnetic resonance imaging of the knee joint were evaluated. Most common presenting symptoms were knee joint pain either acute or long standing 
and swelling. The most common age group to be involved was between 21-29 years. The following patterns of knee injuries were seen:

1. Most common injury was ACL tear of which complete tears were more common. Posterior cruciate ligament tears were less common.

2. Among the Meniscal injuries medial Meniscal tears are more common than lateral meniscus and grade 2 tears are more common in both.

3. Medial collateral ligament tears outnumbered Lateral ligament tears and grade 1 and 2 tears are more common in MCL.

4. Osseous/Osteochondral lesions were seen in 17 patients (34\%). Most of these were bony contusions involving the femoral and tibial condyles.

5. Other abnormalities like discoid meniscus (1), hemarthrosis (2), joint effusions (37), and other myotendinous tears were accurately detected and characterized with the help of magnetic resonance imaging.

CONCLUSION: MRI is an excellent, non-invasive, radiation free imaging modality with multilane capabilities and excellent soft tissue delineation. It can accurately detect, localize and characterize various internal derangements of the knee joint and help in arriving at a correct anatomical diagnosis thereby guiding further management of the patient.

\section{BIBLIOGRAPHY:}

1. DW Polly, JJ Callaghan, RA Sikes, JM McCabe, K McMahon and CG Savory. The accuracy of selective Magnetic resonance imaging compared with findings of Arthroscopy of the knee J Bone Joint Surg 1988; 70:192-198

2. Vaz CES , Camargo OP de, Santana PJ de, Valezi AC. Accuracy of magnetic resonance in identifying traumatic intraarticular knee lesions Clinics 2005; 60(6): 445-50

3. Keith Winters, Russell Tregonning. Reliability of magnetic resonance imaging for traumatic injury of the knee. NZMJ (serial online) 2004 Feb (cited 2005 Feb. 11) ; 118 (1): (1209 screen)

4. Edwin H G Oei, Jeroen J Nikken, Antonio CM Verstijnen, Abida Z Ginai. MR imaging of the menisci and cruciate ligaments. A systematic review Radiology 2003; 226: 837-848

5. Edwin H G Oei, Abida Z Ginai, MG Myriam Hunink. MRI for traumatic knee injury. A Review Semin Ultrasound CT MRI 2007; 28:141-157.

6. Singh JP,Garg L, Shirmali R, Setia V, Gupta V MR Imaging of knee with arthroscopic correlation in twisting injuries. Indian J Radio Imaging 2004; 14: 33-40

7. Thomas H Berquist Osseous and Myotendinous injuries about the knee Radiol Clin N A 2007; 45:955-968 


\section{ORIGINAL ARTICLE}

\begin{tabular}{|c|c|c|}
\hline Age in years & Number & Percentage \\
\hline Up to 19 & 4 & 8.0 \\
\hline $20-29$ & 21 & 42.0 \\
\hline $30-39$ & 17 & 34.0 \\
\hline $40-49$ & 5 & 10.0 \\
\hline$>50$ & 3 & 6.0 \\
\hline Total & 50 & 100.0 \\
\hline
\end{tabular}

\begin{tabular}{|l|c|c|}
\hline \multicolumn{1}{|c|}{ Duration of injury } & Number & Percentage \\
\hline Up to 1 week & 33 & 66.0 \\
\hline 1 week - 2 weeks & 8 & 16.0 \\
\hline 2 weeks - 3 weeks & 6 & 12.0 \\
\hline 3 weeks - 4 weeks & 1 & 2.0 \\
\hline 1 month - 6 months & 2 & 4.0 \\
\hline >6 months & 0 & 0 \\
\hline \multicolumn{1}{|c|}{ Total } & 50 & 100.0 \\
\hline
\end{tabular}

OF PATIENTS STUDIED

TABLE 2: DURATION OF INJURY OF PATIENTS STUDIED

\begin{tabular}{|c|c|c|}
\hline Number & Joint effusion & Percentage \\
\hline No & 13 & 26.0 \\
\hline Yes & 37 & 74.0 \\
\hline \multicolumn{2}{|c|}{ TABLE 3: JOINT EFFUSION } \\
\cline { 2 - 3 }
\end{tabular}

\begin{tabular}{|c|c|c|}
\hline Number & PCL tear & Percentage \\
\hline No & 46 & 92 \\
\hline Yes & 4 & 8 \\
\hline \multicolumn{2}{|r|}{ TABLE 5: PCL TEAR } \\
\hline
\end{tabular}

\begin{tabular}{|c|c|c|}
\hline Number & LCL tear & Percentage \\
\hline No & 46 & 92 \\
\hline Yes & 4 & 8 \\
\hline Grade I & 3 & 75 \\
\hline Grade II & 1 & 25 \\
\hline Grade III & 0 & 0 \\
\hline \multicolumn{2}{|c|}{ TABLE 7: LCL TEAR } \\
\hline
\end{tabular}

\begin{tabular}{|c|c|c|}
\hline LM tear & Number & Percentage \\
\hline No & 41 & 82 \\
\hline Yes & 9 & 18 \\
\hline Grade I & 3 & 33.3 \\
\hline Grade II & 6 & 66.6 \\
\hline Grade III & 0 & 0 \\
\hline
\end{tabular}

\begin{tabular}{|c|c|c|}
\hline Number & ACL tear & Percentage \\
\hline No & 28 & 56 \\
\hline Yes & 22 & 44 \\
\hline Partial & 7 & 31 \\
\hline Complete & 15 & 68 \\
\hline
\end{tabular}

TABLE 4: ACL TEAR

\begin{tabular}{|c|c|c|}
\hline Number & MCL tear & Percentage \\
\hline No & 31 & 62 \\
\hline Yes & 19 & 38 \\
\hline Grade I & 7 & 36 \\
\hline Grade II & 7 & 36 \\
\hline Grade III & 5 & 26 \\
\hline \multicolumn{2}{|c|}{ TABLE 6: MCL TEAR } \\
\hline
\end{tabular}

\begin{tabular}{|c|c|c|}
\hline MM tear & Number & Percentage \\
\hline No & 31 & 62 \\
\hline Yes & 19 & 38 \\
\hline Grade I & 6 & 31.5 \\
\hline Grade II & 10 & 52.6 \\
\hline Grade III & 3 & 15.7 \\
\hline \multicolumn{2}{|c|}{ TABLE 8: MM TEAR } \\
\hline
\end{tabular}

TABLE 9: LM TEAR

\begin{tabular}{|c|c|c|}
\hline $\begin{array}{c}\text { Osseous/Osteochondral } \\
\text { lesion }\end{array}$ & Number & Percentage \\
\hline No & 33 & 66 \\
\hline Yes & 17 & 34 \\
\hline TABLE 10: OSSEOUS/OSTEOCHONDRAL LESIONS
\end{tabular}




\section{ORIGINAL ARTICLE}

\begin{tabular}{|c|c|c|}
\hline MRI findings & Positive findings & $\%$ \\
\hline Joint effusion & 37 & 74 \\
\hline ACL tear & 22 & 44 \\
\hline PCL tear & 4 & 8 \\
\hline MCL tear & 19 & 38 \\
\hline LCL tear & 4 & 8 \\
\hline MM tear & 19 & 38 \\
\hline LM tear & 9 & 18 \\
\hline $\begin{array}{c}\text { Osseous/Osteochondral } \\
\text { lesions }\end{array}$ & 17 & 34 \\
\hline
\end{tabular}

\begin{tabular}{|c|c|c|}
\hline Arthroscopic/surgical findings & No. & $\%$ \\
\hline Not available & 30 & 60 \\
\hline Available & 20 & 40 \\
\hline
\end{tabular}

\section{TABLE 12: ARTHROSCOPIC/SURGICAL FINDINGS}

\begin{tabular}{|c|c|c|}
\hline Correlation & Number & Percentage \\
\hline Yes & 17 & 85 \\
\hline No & 3 & 15 \\
\hline
\end{tabular}

TABLE 13: CORRELATION OF MRI DIAGNOSIS

WITH ARTHROSCOPIC/SURGICAL FINDINGS

TABLE 11: SPECTRUM OF MRI FINDINGS

\begin{tabular}{|c|c|c|}
\hline Combined/Multiple injuries & Number & Percentage \\
\hline Isolated & 21 & 42 \\
\hline Two injuries & 16 & 32 \\
\hline Three injuries & 10 & 20 \\
\hline Four injuries & 3 & 6 \\
\hline Five injuries & 0 & 0 \\
\hline TABLE 14: COMBINED / MULTIPLE INJURIES
\end{tabular}

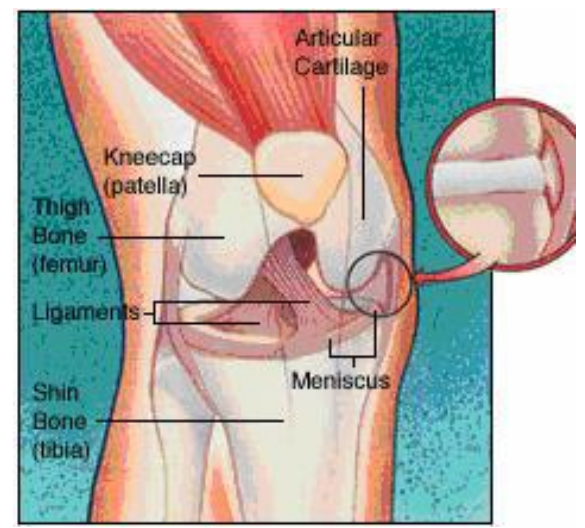

FIGURE-1 NORMAL KNEE JOINT

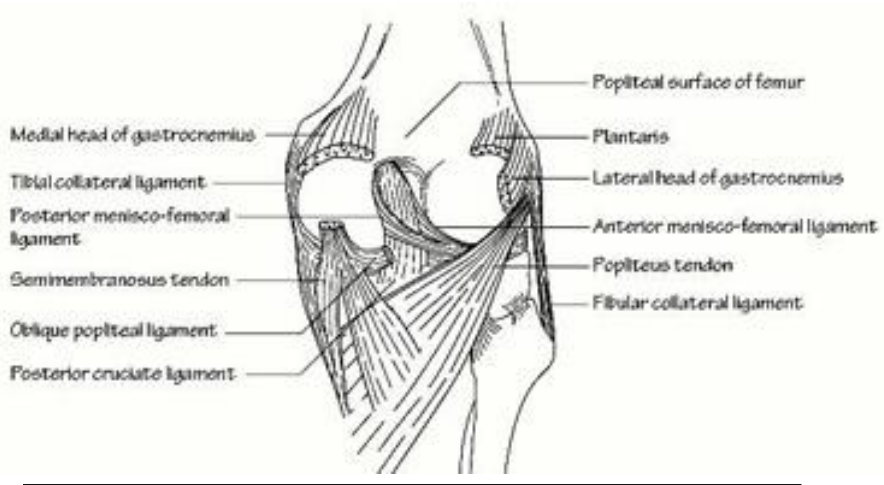

FIGURE 2 EXTRA CAPSULAR LIGAMENTS 


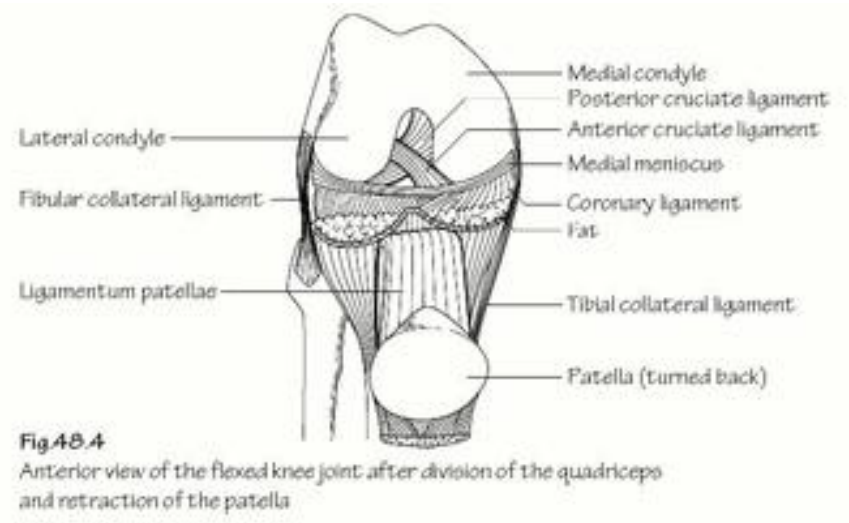

\section{FIGURE 3 ANTERIOR VIEW OF THE FLEXED KNEE JOINT \\ AFTER DIVISION OF THE QUADRICEPS AND RETRACTION OF THE PATELLA}

\section{AUTHORS:}

1. Rangappa Hanumappa Chavadaki

2. Praveen Kumar Reddy P.

3. Reneesh Uruniyan Paramban

4. Swetha Gurram

\section{PARTICULARS OF CONTRIBUTORS:}

1. Associate Professor, Department of Orthopedics, Navodaya Medical College Hospital \& Research Centre, Raichur.

2. Assistant Professor, Department of Orthopedics, Navodaya Medical College Hospital \& Research Centre, Raichur.

3. Senior Resident, Department of Orthopedics, Navodaya Medical College Hospital \& Research Centre, Raichur.
4. Senior Resident, Department of Orthopedics, Navodaya Medical College Hospital \& Research Centre, Raichur.

\section{NAME ADDRESS EMAIL ID OF THE CORRESPONDING AUTHOR:}

Dr. Reneesh Uruniyan Paramban, Senior Resident, Department of Orthopedics, Navoday a Medical College Hospital \& Research Centre, Raichur

Email -drreneesh@gmail.com

Date of Submission: 03/10/2013.

Date of Peer Review: 04/10/2013.

Date of Acceptance: 08/10/2013.

Date of Publishing: 21/10/2013 